\title{
Evaluation of the common variants of the ABCA4 gene in families with Stargardt disease and autosomal recessive retinitis pigmentosa
}

\author{
BARKUR S. SHASTRY \\ Department of Biological Sciences, Oakland University, Rochester, MI 48309, USA \\ Received October 30, 2007; Accepted January 7, 2008
}

\begin{abstract}
Stargardt disease (STGD) is one of the most common autosomal recessive retinal dystrophies with an estimated incidence of one in 10,000. It affects the central retina (macula). Retinitis pigmentosa (RP) comprises a large and exceptionally heterogeneous group of hereditary disorders of the retina. It is caused by the loss of photoreceptors. The condition is a degenerative disorder characterized by retinal pigment deposits and has an estimated incidence of one in 4,000. Although, to date, 45 known loci have been identified, none of them independently account for a substantial portion of RP. Recently, the photoreceptor cell-specific ATP-binding cassette transporter (ABCA4) gene was found to be mutated in patients with STGD as well as autosomal recessive RP. In order to further understand the contribution of this gene to the susceptibility to STGD and RP, we analyzed three unrelated STGD families and one autosomal recessive RP family specifically for the more common variants (A1038V, G1961E, $2588 \mathrm{G} \rightarrow \mathrm{C}, \mathrm{R} 943 \mathrm{Q}$ or $2828 \mathrm{G} \rightarrow \mathrm{A}$ ) in the ABCA4 gene. Our analyses employing standard techniques such as polymerase chain reaction, restriction fragment length polymorphism, and direct DNA sequencing of amplified products were able to identify one common variant (R943Q) in all three STGD families but not in the RP family. All three affected STGD individuals, however, were heterozygous for this variation, and this alteration did not segregate with the disease and was also present in the normal controls. Similar analysis of other common variants revealed no pathogenic mutations in the STGD and RP families. It is likely that the variant identified in this study represents a rare polymorphism (non-pathogenic). Although, at present we cannot eliminate the possibility of this gene as a candidate gene, future extensive studies on this as well as other candidate genes may uncover the susceptibility
\end{abstract}

Correspondence to: Dr Barkur S. Shastry, Department of Biological Sciences, Oakland University, Rochester, MI 48309, USA

E-mail: shastry@oakland.edu

Key words: amplification, autosome, disease, gene, mutation, polymerase, variant gene for these recessive forms of the disorders in these families.

\section{Introduction}

Stargardt disease (STGD), which is also known as juvenile macular degeneration, was first reported by Dr Stargardt in 1909. It is one of the most common hereditary retinal dystrophies with an estimated incidence of one in 10,000 and accounts for $\sim 7 \%$ of all retinal dystrophies. The condition affects the central retina (macula) with a variable phenotype and a variable age of onset and severity. Most cases present with central vision loss with onset in childhood and young adulthood. Both sexes are equally affected. The disorder is characterized by progressive bilateral macular atrophy with characteristic orange-yellow flecks in the macular and the perimacular region resulting in central vision impairment. Histologically, STGD is associated with a massive deposition of lipofuscin-like material in the retinal pigment epithelium which has also been observed in aging human eyes. The disorder is clinically similar to fundus flavimaculatus (FFM) which has a later age of onset and slower progression or milder visual loss. Both of these disorders in most cases are inherited in an autosomal recessive mode but there have been a number of cases that have been identified as autosomal dominant STGD with juvenile onset of macular degeneration $(1,2)$. It has been suggested from genetic analysis that both STGD and FFM are allelic disorders with a slightly different clinical manifestation.

Linkage analysis has mapped the STGD gene on chromosome 1p13-p21, and a gene (ABCA4) encoding a retina-specific ATP-binding transmembrane transporter protein was isolated (3). ABCA4 is a large gene consisting of 50 exons and codes for a 2,273 amino acid protein. It is localized to the rims of the rod and cone outer segment disks by in situ hybridization. This protein is involved in the energydependent transport of substrates across the membrane. Several series of mutational analyses (3-5) confirm that ABCA4 is the principal gene causing STGD. Additionally, the same gene is found to be mutated in FFM $(4,5)$, retinitis pigmentosa (6), cone-rod dystrophy (6) and in certain cases of age-related macular degeneration (7). All of these mutational analyses show a broad spectrum of mutations and allelic and non-allelic heterogeneity in a minority of families suggesting that there is at least one additional minor STGD locus. 
Notably, it has also been found that certain mutant alleles are more common in certain populations than others. Recently, researchers have also developed a mouse model of STGD by knocking-out the ABCA4 gene. These mice exhibit many characteristics of STGD such as cone cell death and lipofuscin accumulation (8), and these mice will be useful for the development of drugs to cure, prevent or delay the progression of this disorder.

Retinitis pigmentosa (RP) is a group of progressive pigmentary retinopathies. It is caused by the loss of photoreceptors and characterized by retinal pigment deposits predominantly in the peripheral retina. As it first involves degeneration of rods and then cone photoreceptors, it is also known as rod-cone dystrophy. The disorder affects both eyes, and the affected individuals in the early stage experience night blindness. As the disease progresses, loss of central vision occurs resulting in complete loss of sight (9). In most cases, RP is non-syndromic but there are other syndromic forms such as Usher syndrome. It is a clinically and genetically heterogeneous disorder and has an estimated prevalence of one in 4,000. It can be inherited as an autosomal dominant (AD), autosomal recessive (AR) and X-linked (XL) recessive trait (reviewed in ref. 10). Of these, the AR form is considered to be the most common form of RP worldwide. To date, there are $15 \mathrm{AD}, 24 \mathrm{AR}$ and $5 \mathrm{XL}$ loci that have been identified. In many cases the corresponding genes and their mutations have also been studied (reviewed in ref. 11). As a result of these extensive studies, the signaling pathway leading to the degeneration of photoreceptors has been described (reviewed in ref. 12) in some forms of the disorder. However, none of the above loci independently account for a significant portion of any one form of inheritance. Considering all of the available data, cloned genes account for $\sim 80 \%$ of XL, $50 \%$ of $\mathrm{AD}$ and $40 \%$ of AR forms. This suggests that there are many more genes that still remain to be identified (9). To further extend the diagnostic and prognostic value of the molecular genetic study of STGD and to understand the genetic heterogeneity of RP, in this report we analyzed three unrelated STGD families and one AR RP family specifically for the more common variants (A1038V, G1961E, R943Q or $2828 \mathrm{G} \rightarrow \mathrm{A}$ and $2588 \mathrm{G} \rightarrow \mathrm{C}$ ) of the ABCA4 gene which when mutated produce a broad spectrum of the retinal phenotypes including RP and age-related macular degeneration (AMD). Additionally, we also analyzed the common variant (Y402H) of the complement factor $\mathrm{H}(\mathrm{CFH})$ gene for its contribution to the RP phenotype since some family members exhibited AMD.

\section{Materials and methods}

Patient studies. Three small STGD families with four affected individuals were identified. Ocular examination of all patients by an experienced ophthalmologist revealed very strong clinical features of STGD (Fig. 1) including peripheral pigmented lesions (individual I-3 and II-1 in pedigrees 1 and 3 respectively). Individuals II-1 (pedigrees 1 and 2) had yellow-white flecked retinas with flecks extending around the posterior pole with pisciform shape. The symptoms in all three families began in the first decade of life (5-8 years) with impaired vision. All three families were diagnosed as having acute STGD. Detailed medical and family histories were obtained from each available member, and a pedigree was constructed (Fig. 1). Due to the small size of the families, the mode of inheritance was unable to be determined, but all of them could have been autosomal recessive families. To the best of our knowledge there was no consanguinity involved in these families.

One family with AR RP was identified through the proband. When the proband was 5 months old she presented with fine intermittent horizontal nystagmus which was associated with head bobbing. A careful examination showed a visual acuity of 20/30. She also had a significant bilateral hyperopia and astigmatism. She had no other developmental problems, and the optic nerve tissue appeared healthy and had good foveal reflexes. When she was 3 and a half years of age her parents noticed that her night vision had decreased dramatically. Subsequent ophthalmological examination revealed peripheral retinal degeneration leading to the diagnosis of RP. Her best-corrected vision was 20/60. Detailed medical and family histories were obtained from each available member and a pedigree was constructed (Fig. 2). Notably, the cousin of the proband at the age of 2 years also presented with nystagmus with head bobbing and then developed night blindness at the age of 4 which later led to the diagnosis of RP. When the pedigree was constructed, family eye history suggested RP as well as age-related macular degeneration (AMD) in the great grandparents and grandparents from both paternal and maternal sides. To the best of our knowledge there was no consanguineous marriage in the family. Additionally, we could not verify the medical records of deceased individuals, but this information was cross-checked with several family members. The Institutional Review Board of Oakland University approved the study, and all families were informed of the purpose of the study.

Sample collection and PCR amplification. Venous blood was collected from patients and normal individuals, and leukocyte DNA was extracted. Genomic DNA from normal and affected individuals was amplified by the polymerase chain reaction (PCR) with commercially synthesized primers described previously $(3,13-15)$. The PCR conditions were 30 cycles of $1.5 \mathrm{~min}$ at $94^{\circ} \mathrm{C}, 1 \mathrm{~min}$ at $60^{\circ} \mathrm{C}$ and $2 \mathrm{~min}$ at $72^{\circ} \mathrm{C}$ in a FailSafe PCR buffer system supplied by Epicenter (Madison, WI, USA).

Restriction analysis and sequencing. The amplified product was subjected to restriction enzyme digestion with either 10 units of Alu I $(2588 \mathrm{G} \rightarrow \mathrm{C}$ mutation in exon 17 of ABCA4 gene creates a site for Alu I), or Bse YI (A1038V mutation in exon 21 destroys a site for Bse YI), or Taq I (G1961E variation in exon 42 in ABCA4 creates a Taq I site), or Msp I (R943Q alteration in exon 19 destroys the Msp I site), or Nla III (Y402H mutation in exon 9 of the $\mathrm{CFH}$ gene creates a Nla III site) at $37^{\circ} \mathrm{C}$ (Alu I, Bse YI, Msp I, Nla III) and $65^{\circ} \mathrm{C}$ (Taq I) for $1 \mathrm{~h}$. The digested products were analyzed on a $9 \%$ PAG with Tris-borate-EDTA buffer followed by ethidium bromide staining. The base change in the restriction site was further confirmed by a BigDye terminator cycle sequencing (both strands were sequenced) of the amplified products according to the procedure provided by the manufacturer (Applied Biosystems, Foster City, CA, USA). 
A

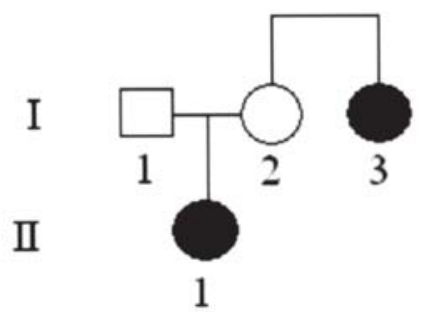

B
2

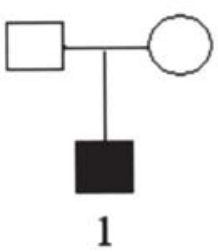

3

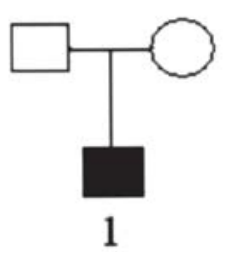

C
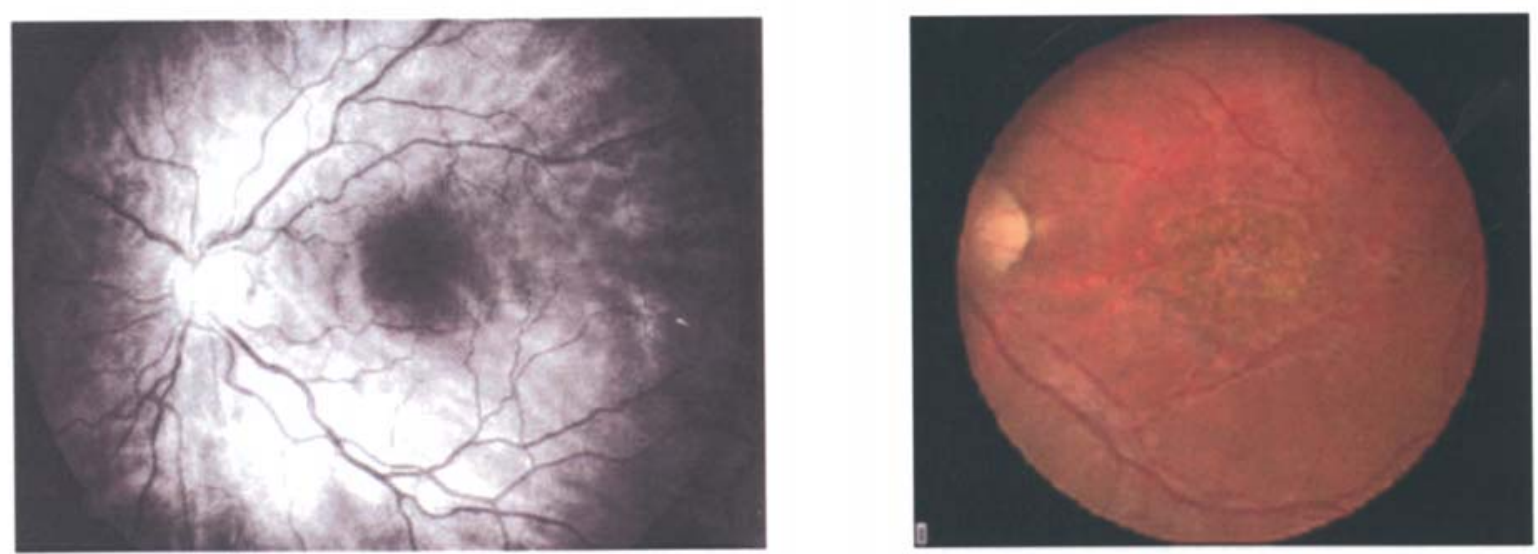

Figure 1. Three STGD families used in this report are shown in A. Filled and open symbols represent affected and unaffected individuals, respectively. The fundus images of a normal (B) and unrelated STGD patient (C) displaying orange-yellow flecks in the macular area.

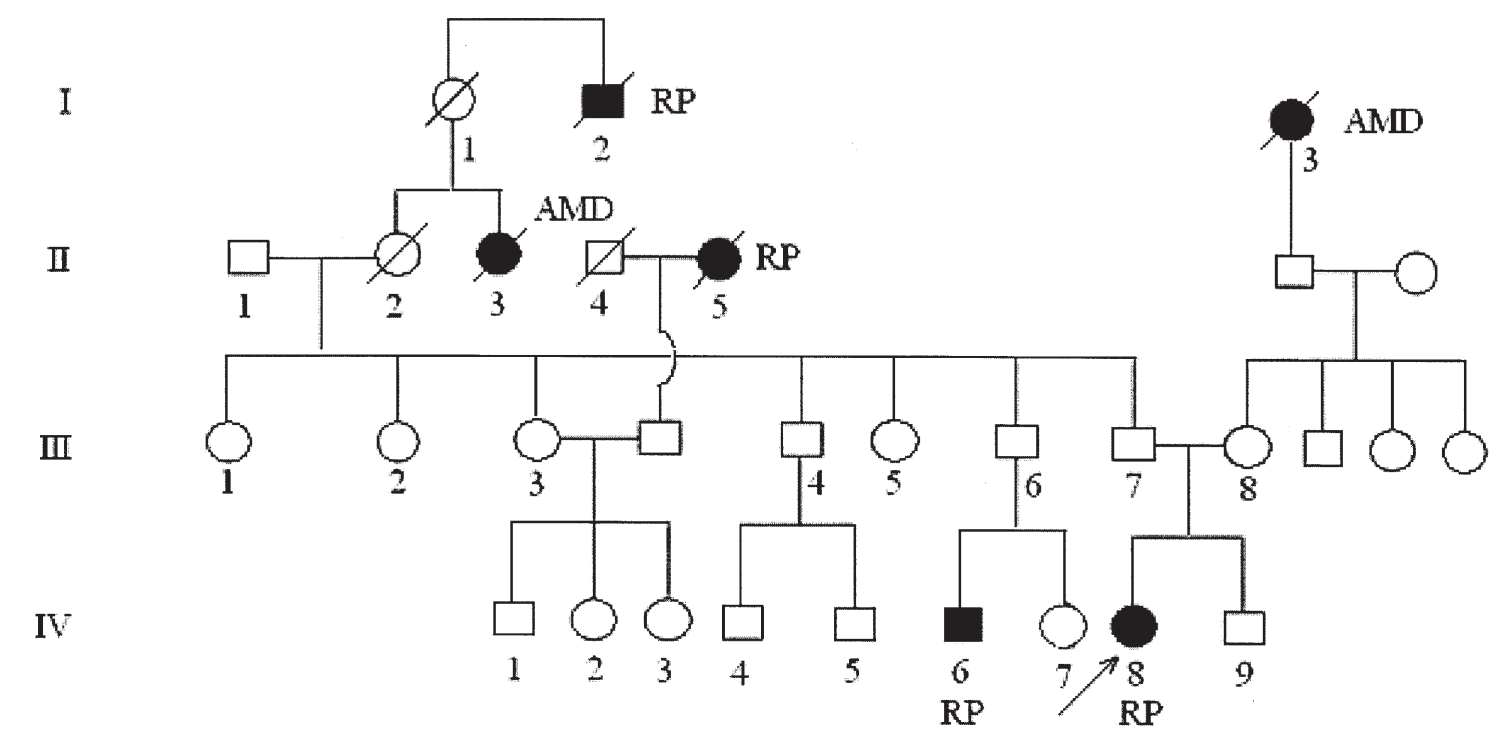

Figure 2. Pedigree of the AR RP family used in this report. Filled and open symbols represent affected and unaffected individuals, respectively. The proband is denoted by an arrow.

\section{Results}

As families were individually too small for statistically significant linkage analysis and due to the fact that the analysis of the STGD gene in its entirety represents a challenging task for any laboratory because of its large size, we followed the approach of the International ABCR Screening Consortium. The finding that the variants A1038V (exon 21), G1961E (exon 42), 2588G $\rightarrow \mathrm{C}$ (exon 17) and R943Q (exon 19) which is previously known as $2828 \mathrm{G} \rightarrow \mathrm{A}(4)$ are more common 
A

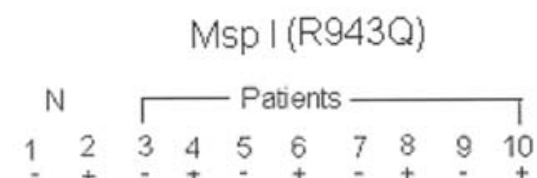

B

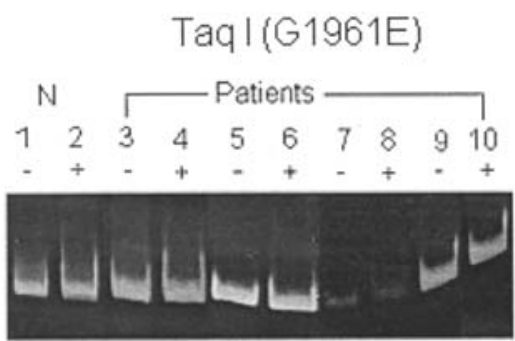

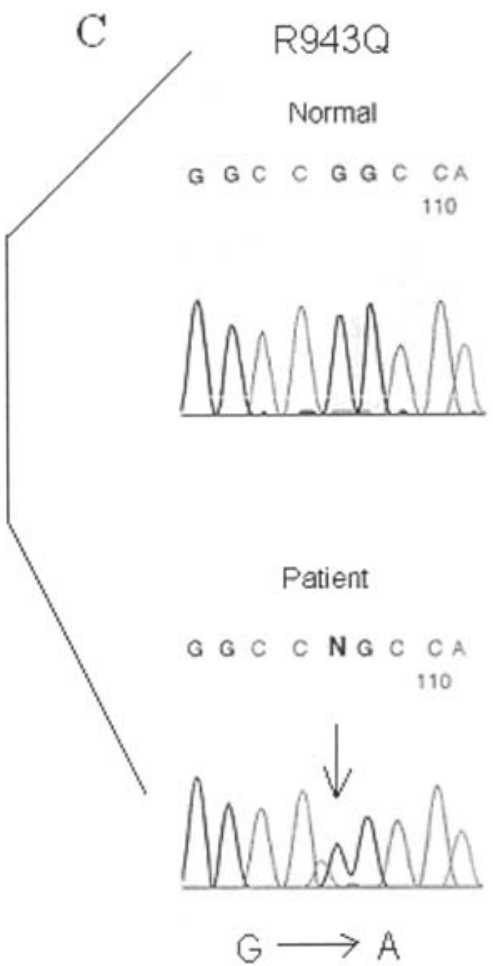

Figure 3. Restriction digestion patterns of PCR-amplified products for R943Q (A) and G1961E (B) variants. All three patients (patients II-1 in families 1-3) were heterozygous (lanes 3-8 in A) for the R943Q mutation whereas the affected individual I-3 in family 1 was completely normal (lanes 9-10). Similar experiments with Taq I enzyme for the G1961E mutation did not detect any alterations (B). The destruction of the Msp I site was further confirmed by DNA sequence analysis that identified the variant $\mathrm{G} \rightarrow \mathrm{A}$ in the Msp I site (C). Symbols (-) and (+) indicate undigested and digested PCR fragments, respectively.

A

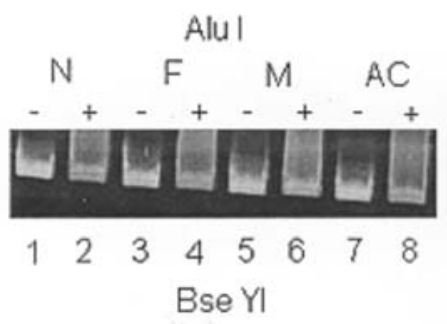

B

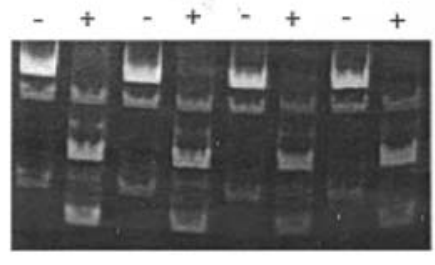

$\begin{array}{llllllll}1 & 2 & 3 & 4 & 5 & 6 & 7 & 8\end{array}$

Taq I

C

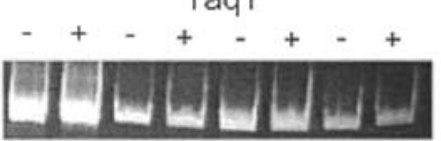

$\begin{array}{llllllll}1 & 2 & 3 & 4 & 5 & 6 & 7 & 8\end{array}$

Nla III

D

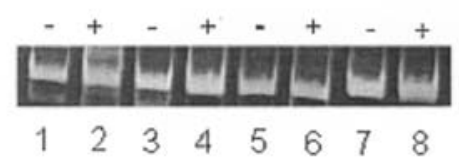

$\mathrm{ABCA} 4-$ Exon 17 $2588 \mathrm{G} \rightarrow \mathrm{C}$

ABCA4- Exon 21

$\mathrm{A} 1038 \mathrm{~V}$

ABCA4- Exon 42 G1961E

\section{CFH- Exon 9 \\ $\mathrm{Y} 402 \mathrm{H}$}

Figure 4. Restriction digestion patterns of PCR-amplified products for variants $2588 \mathrm{G} \rightarrow \mathrm{C}(\mathrm{A}), \mathrm{A} 1038 \mathrm{~V}$ (B), G1961E (C) and Y402H (D). Symbols $(-)$ and $(+)$ indicate undigested and digested PCR fragments, respectively. $\mathrm{N}$, normal; F, father; $\mathrm{M}$, mother; and AC, affected child. alterations which predispose individuals to STGD, prompted us to further evaluate these variants for their contribution to the susceptibility to STGD in additional small families. The restriction enzyme digestion patterns for R943Q (Msp I) and G1961E (Taq I) changes are shown in Fig. 3A and B respectively. As expected, the amplified fragment from the normal individual was completely cleaved (Fig. 3A, lanes 1 and 2) by the enzyme Msp I. However, DNAs from patients were only partially cleaved (lanes 3-8; families 1, 2, 3, patients II-1) except in patient I-3 in family 1 (lanes 9-10) where the amplified product was completely cleaved. This suggests that the Msp I site was mutated in one of the alleles in three of four patients. The destruction of the Msp I site was subsequently confirmed by DNA sequence analysis which identified the variant $(\mathrm{G} \rightarrow \mathrm{A})$ in the Msp I site (Fig. 3C) as expected. This experiment demonstrated that three out of four patients were heterozygous for the R943Q alteration, but this mutation did not segregate in the family (family 1). Similar experiments with the Taq I enzyme for the G1961E mutation detected no alteration in any patient (Fig. 3B). Additionally, we failed to detect the other two alterations (A1038V and $2588 \mathrm{G} \rightarrow \mathrm{C}$ ).

One family with AR RP was identified through the proband. Since the family was too small for statistically significant linkage analysis and linkage studies of AR disorders in consanguineous families are mainly based on homozygosity mapping and this method cannot be applied in the case of non-consanguineous families, we employed a candidate gene approach in this study. Based on the fact that mutations in the ABCA4 gene can cause a spectrum of retinal phenotypes including AR RP and AMD (6,16-18), we 
selected this gene as a candidate gene for this family. However, analysis of the ABCA4 gene in its entirety represents a challenging task for any laboratory since it contains 50 exons. Therefore, we followed the approach of the International ABCR Screening Consortium, specifically analyzing for the common variants. Additionally, variants G1961E in the ABCA4 gene and Y402H in exon 9 of the $\mathrm{CFH}$ gene are associated with AMD at a statistically significant level. These observations prompted us to further evaluate these variants for their contribution to AR RP in an additional small family shown in Fig. 2. The restriction enzyme digestion patterns are shown in Fig. 4. As can be observed from Fig. 4A-D, mutation neither introduced new (A, C and D) nor destroyed existing (B) restriction sites. The digestion patterns were identical (lanes 1 and 2 versus lanes 3-8 in each panel) between the normal and affected individual in the amplified fragments. This suggests that the above four common alterations were not present and not involved in the disease pathology of the family. It remains to be seen whether any other exons of the ABCA4 gene have alterations, or a completely different gene is involved in the pathogenesis of the disorder.

\section{Discussion}

To further extend the diagnostic and prognostic value of the molecular genetic study of STGD and AR RP, in this report we analyzed three unrelated STGD families and one AR RP family specifically for the more common variants for their contribution to the susceptibility to the above disorders by employing standard techniques such as PCR, RFLP and DNA sequencing. The International ABCR Consortium has recently used this approach of analyzing the more common variants because the analysis of the ABCA4 gene in its entirety represents a challenging task due to its large size. Additionally, such analysis of common variants has been recently successively employed by several investigators to show that variants in the CFH $(\mathrm{Y} 402 \mathrm{H})$ and LOC 387715 (A69S) genes are strongly associated with age-related macular degeneration and account for a major fraction of the disorder. Therefore, the information obtained by using this approach in the present study is still valuable. Our analysis detected only one of the more common variants R943Q (also known as $2828 \mathrm{G} \rightarrow \mathrm{A}$ ) located in exon 19 in all three STGD families (but not in RP), and three of the four affected individuals were heterozygous for this mutation. The R943Q alteration results in a non-conservative amino acid substitution, and this variation was most often found to be in linkage disequilibrium with presumably a pathogenic variation $2588 \mathrm{G} \rightarrow \mathrm{C}$ in exon 17 in a western European population (4). In the present study, however, we did not find the co-occurrence of these two variants which could be due to the lower frequency of $2588 \mathrm{G} \rightarrow \mathrm{C}$ in control $(0.9 \%)$ and in patients $(8.7 \%)$ with STGD in the US (3). Additionally, as suggested by others, the R943Q variation may not be a pathogenic mutation by itself because it was not segregating in the family (STGD family 1), and the affected individual (I-3) had normal alleles (Fig. 3A, lanes 9-10). Moreover, it was also found to be present in control populations without the $2588 \mathrm{G} \rightarrow \mathrm{C}$ mutation with a frequency of $5.8 \%(3,4)$. Analysis of other exons also revealed no additional common mutations in these three families as well as in the RP family. Failure to identify pathogenic mutations in the above study was not surprising because mutational analyses of STGD show a broad spectrum of variation and non-allelic and allelic heterogeneity (4). Moreover, our results suggest that the common variants in the ABCA4 gene may be population-specific, and this method of screening is not widely applicable to all populations. This is exemplified by the failure to detect the common variant of the CFH gene in Japanese patients with AMD. Although we cannot completely rule out the ABCA4 gene in these families, there is a possibility that an entirely different gene may be involved. We also cannot assess the functional consequences of the R943Q alteration in the affected individuals at present because there is no functional test for ABCA4 activity. It may act synergistically with other unknown pathological variants, increase the risk of developing STGD or it may simply represent a neutral polymorphism. Identification of pathogenic variants in the ABCA4 gene or other genes (non-allelic heterogeneity) in these families coupled with a functional test for ABCA4 activity may be able to resolve these questions in the future.

\section{Acknowledgements}

I am thankful to Dr Michael T. Trese of William Beaumont Hospital, Royal Oak, MI and Dr Scott D. Pendergast of Retina Association of Cleveland Inc., $\mathrm{OH}$ for providing the STGD and RP families and the fundus picture of the disease, respectively. This work was supported in part by the Oakland University Research Excellence Program in Biotechnology.

\section{References}

1. Edwards AO, Miedziak A, Vrabec T, Verhoeven J, Acott TS, Weleber RG and Donoso LA: Autosomal dominant Stargardtlike macular dystrophy: I. Clinical characterization, longitudinal follow-up, and evidence for a common ancestry in families linked to chromosome 6q14. Am J Ophthalmol 127: 426-435, 1999.

2. Kniazeva M, Chiang MF, Morgan B, Anduze AL, Zack DJ, Han $\mathrm{M}$ and Zhang $\mathrm{K}$ : A new locus for autosomal dominant Stargardt-like disease maps to chromosome 4. Am J Hum Genet 64: 1394-1399, 1999.

3. Allikmets R, Singh N, Sun H, Shroyer NF, Hutchinson A, Chidambaram A, Gerrard B, Baird L, Stauffer D, Peiffer A, Rattner A, Smallwood P, Li Y, Anderson KL, Lewis RA, Nathans J, Leppert M, Dean M and Lupski JR: A photoreceptor cell-specific ATP binding transporter gene (ABCR) is mutated in recessive Stargardt macular dystrophy. Nat Genet 15: 236-246, 1997.

4. Maugeri A, van Driel MA, van de Pol DJ, Klevering BJ, van Haren FJ, Tijmes N, Bergen AA, Rohrschneider K, Blankenagel A, Pinckers AJ, Dahl N, Brunner HG, Deutman AF, Hoyng CB and Cremers FP: The $2588 \mathrm{G} \rightarrow \mathrm{C}$ mutation in the $\mathrm{ABCR}$ gene is a mild frequent founder mutation in the western European population and allows the classification of ABCR mutations in patients with Stargardt disease. Am J Hum Genet 64: 1024-1035, 1999.

5. Rozet J-M, Gerber S, Souied E, Perrault I, Chatelin S, Ghazi I, Leowski C, Dufier J-L, Munnich A and Kaplan J: Spectrum of ABCR gene mutations in autosomal recessive macular dystrophies. Eur J Hum Genet 6: 291-295, 1998.

6. Cremers FP, van de Pol DJ, van Driel M, den Hollander AI, van Haren FJ, Knoers NV, Tijmes N, Bergen AA, Rohrschneider K, Blankenagel A, Pinckers AJ, Deutman AF and Hoyng CB: Autosomal recessive retinitis pigmentosa and cone-rod dystrophy caused by splice site mutations in the Stargardt disease gene ABCR. Hum Mol Genet 7: 355-362, 1998. 
7. Allikmets R, Shroyer NP, Singh N, Seddon JM, Lewis RA, Bernstein PS, Peiffer A, Zabriskie NA, Li Y, Hutchinson A, Dean M, Lupski JR and Leppert M: Mutation of the Stargardt disease gene (ABCR) in age-related macular degeneration. Science 277: 1805-1807, 1997.

8. Weng J, Mata NL, Azarian SM, Tzekov RT, Birch DG and Travis GH: Insights into the function of rim protein in photoreceptors and etiology of Stargardt's disease from the phenotype in abcr knockout mice. Cell 98: 13-23, 1999.

9. Hamel C: Retinitis pigmentosa. Orphanet J Rare Dis 1: 40-52, 2006.

10. Shastry BS: Hereditary degenerative retinopathies: optimism for somatic gene therapy. IUBMB Life 49: 479-484, 2000.

11. Shastry BS: Retinitis pigmentosa and related disorders Am J Med Genet 52: 467-474, 1994.

12. Shastry BS: Signal transduction in the retina and inherited retinopathies. Cell Mol Life Sci 53: 419-429, 1997.

13. Gerber S, Rozet JM, van de Pol TJR, Hoyng CB, Munnich A, Blankenagel A, Kaplan J and Cremers FPM: Complete exonintron structure of the retina-specific ATP binding transporter gene (ABCR) allows the identification of novel mutations underlying Stargardt disease. Genomics 48: 139-142, 1998.
14. Rivera A, White K, Stohr H, Steiner K, Hemmrich N, Grimm T, Jurklies B, Lorenz B, Scholl HPN, Apfelstedt-Sylla E and Weber BHF: A comprehensive survey of sequence variation in the ABCA4 (ABCR) gene in Stargardt disease and age-related macular degeneration. Am J Hum Genet 67: 800-813, 2000.

15. Haines JL, Hauser MA, Schmidt S, Scott WK, Olmson LM, Gallins P, Spencer K, Kwan SY, Noureddine M, Gilbert JR, Schnetz-Boutaud N, Agarwal A, Postel EA and Pericak-Vance MA: Complement factor $\mathrm{H}$ variant increases the risk of age-related macular degeneration. Science 308: 419-422, 2005.

16. Klevering BJ, Deutman AF, Maugeri A, Cremeres FPM and Hoyng CB: The spectrum of retinal phenotypes caused by mutations in the ABCA4 gene. Graefes Arch Clin Exp Ophthalmol 243: 90-100, 2005.

17. Martinez-Mir A, Paloma E, Allikmets R, Ayuso C, del Rio T, Dean M, Vilageliu L, Gonzalez-Duarte R and Balcells S: Retinitis pigmentosa caused by a homozygous mutation in the Stargardt disease gene ABCR. Nat Genet 18: 11-12, 1998.

18. Allikmets $\mathrm{R}$ and The International ABCR Screening Consortium: Further evidence for an association of ABCR alleles with age-related macular degeneration. Am J Hum Genet 67: 487-491, 2000. 